\title{
A randomised, open-label study of umeclidinium versus glycopyrronium in patients with COPD
}

\author{
Tara Rheault ${ }^{1}$, Sanjeev Khindri ${ }^{2}$, Mitra Vahdati-Bolouri ${ }^{2}$, Alison Church $^{1}$ and \\ William A. Fahy ${ }^{2}$
}

Affiliations: ${ }^{1}$ GSK, Respiratory and Immuno-Inflammation, Research Triangle Park, NC, USA. ${ }^{2}$ GSK, Respiratory Research and Development, Middlesex, UK.

Correspondence: Tara Rheault, Quintiles, 5827 S. Miami Blvd, Morrisville, NC 27560, USA.

E-mail: Tara.Rheaultaquintiles.com

ABSTRACT This study compared the efficacy and safety of once-daily umeclidinium $62.5 \mu \mathrm{g}$ with oncedaily glycopyrronium $50 \mu \mathrm{g}$ in patients with moderate-to-severe chronic obstructive pulmonary disease.

This was a 12-week, multicentre, randomised, open-label, parallel-group study (Clinicaltrials.gov: NCT02236611). Patients were randomised $1: 1$ to umeclidinium $62.5 \mu \mathrm{g}$ or glycopyrronium $50 \mu \mathrm{g}$ administered via Ellipta or Breezhaler dry powder inhaler, respectively. The primary endpoint was trough forced expiratory volume in $1 \mathrm{~s}(\mathrm{FEV} 1)$ at day 85 in the per-protocol population. Other endpoints included: weighted mean FEV1 over $0-24 \mathrm{~h}$ and patient-reported outcomes (transition dyspnoea index score and St George's Respiratory Questionnaire total score). Adverse events were also assessed.

A total of 1037 patients were randomised to treatment. Umeclidinium was non-inferior (margin: $-50 \mathrm{~mL}$ ) to glycopyrronium (trough $\mathrm{FEV} 1$ at day 85 treatment difference: $24 \mathrm{~mL}$, 95\% confidence intervals: -5-54). Improvements in other endpoints were similar between treatments. Adverse event incidences were similar for umeclidinium (37\%) and glycopyrronium (36\%).

Once-daily umeclidinium was non-inferior to once-daily glycopyrronium in patients with chronic obstructive pulmonary disease in trough $\mathrm{FEV}_{1}$ at day 85. Patient-reported outcomes and safety profiles were similar for both treatments.

@ERSpublications

Once-daily umeclidinium is non-inferior to once-daily glycopyrronium in patients with COPD by trough FEV1 at day $85 \mathrm{http}: / /$ ow.ly/4mRdE6

This article has supplementary material available from openres.ersjournals.com

Received: Dec 162015 | Accepted after revision: March 042016

Clinical trials: This study is registered at clinicaltrials.gov with identifier number NCT02236611

Conflict of interest: Disclosures can be found alongside this article at openres.ersjournals.com

Copyright $\odot$ ERS 2016. This article is open access and distributed under the terms of the Creative Commons Attribution Non-Commercial Licence 4.0. 


\section{Introduction}

Chronic obstructive pulmonary disease (COPD) is characterised by persistent airflow limitation that is not fully reversible post-bronchodilation [1]. Inhaled bronchodilators such as long-acting muscarinic antagonists (LAMAs) are central to the pharmacological management of stable, moderate-to-very-severe COPD [1]. LAMAs used as maintenance treatments to alleviate symptoms in patients with COPD include tiotropium (TIO) [2], glycopyrronium (GLYCO; once or twice daily) [3, 4], umeclidinium (UMEC) $[5,6]$ and twice-daily aclidinium [7, 8]. TIO and UMEC are the only LAMAs currently approved for once-daily use in the USA and EU $[2,5,6,9]$.

Randomised controlled trials directly comparing LAMAs are few in number; however, once-daily GLYCO $50 \mu \mathrm{g}$ has been shown to be non-inferior in terms of lung function to TIO $18 \mu \mathrm{g}$ in the large Glycopyrronium Bromide in Chronic Obstructive Pulmonary Disease Airways clinical study (GLOW 5), 12-week, randomised, blinded, non-inferiority study [10] and in an independent review of all clinical trials comparing these two active treatments alone [11]. In the absence of comparative randomised controlled data indirect treatment comparisons between LAMAs through network meta-analyses have been conducted [12-15]. These studies demonstrated similarity between the LAMAs compared [12-15]; however, UMEC was included in just one of these analyses [14]. In addition, such indirect comparisons can be confounded by study design differences. Therefore, robust direct formal comparisons between LAMAs, including UMEC, are needed.

Administration of once-daily UMEC $62.5 \mu \mathrm{g}$ (delivered dose: $55 \mu \mathrm{g}$ ) via the Ellipta (GlaxoSmithKline, Hertfordshire, UK) dry powder inhaler (DPI) or once-daily GLYCO $50 \mu \mathrm{g}$ (delivered dose: $44 \mu \mathrm{g}$ ) via the Breezhaler (Novartis, Basel, Switzerland) DPIs have yet to be formally compared in any study, although both LAMAs are reported to improve lung function compared with placebo in patients with moderate-to-very-severe COPD [16-20]. Clinically important improvements in dyspnoea, health-related quality of life (HRQoL) and rescue-medication use have also been reported with both LAMAs (UMEC [16, 17], GLYCO [18, 20]).

This study is the first to formally compare the efficacy and safety of once-daily UMEC $62.5 \mu \mathrm{g}$ with once-daily GLYCO $50 \mu \mathrm{g}$ in patients with moderate-to-severe COPD.

\section{Methods}

\section{Study design}

This was a multicentre, randomised, open-label, two-arm, parallel-group study primarily designed to demonstrate that UMEC is non-inferior to GLYCO as measured by the primary endpoint of trough forced expiratory volume in $1 \mathrm{~s}$ (FEV1) after 12 weeks ( 85 days) of treatment in the per-protocol population (GSK study identifier: 201315; Clinicaltrials.gov identifier: NCT02236611). The study was performed between September 2014 and June 2015. All patients provided written informed consent before participating in the study. The study protocol was approved by the relevant ethics committee or institutional review board.

Patients meeting eligibility criteria at screening (visit 1) completed a 7-14-day run-in period before randomisation at visit 2 . The randomisation code was generated by GSK using a validated computerised system RandAll version NG (GlaxoSmithKline) and patients were randomised using the Registration And Medication Ordering System (RAMOS; GlaxoSmithKline) interactive response technology. Eligible patients were randomised to 84 days of treatment, with clinic visits on days 2, 28, 56, 84 and 85 . Randomisation was stratified according to whether or not the patient underwent the 24-h serial FEV1 assessments. The total duration of study participation was approximately 15 weeks.

\section{Patients}

Eligible patients were: $\geqslant 40$ years of age with a diagnosis of COPD according to the American Thoracic Society/European Respiratory Society guidelines [21]; had an $\mathrm{FEV} 1 /$ forced vital capacity (FVC) ratio of $<0.70$ and a post-salbutamol $\mathrm{FEV} 1$ of $\geqslant 30 \%$ and $\leqslant 70 \%$ of predicted normal values at the screening visit; were current or former smokers, who had a history of smoking of $\geqslant 10$ pack-years; and had a modified Medical Research Council dyspnoea score of $\geqslant 2$ at the screening visit.

The key exclusion criteria included: current diagnosis of asthma or other respiratory disorders; hospitalisation for COPD or pneumonia within 12 weeks before the screening visit; lung volume reduction surgery 12 months before this visit; or long-term oxygen use. Use of other COPD maintenance medications, antibiotics or systemic corticosteroids was not permitted before enrolment within designated time intervals (supplementary table s1).

The additional criteria for randomisation were patients must not have experienced a COPD exacerbation during the run-in period or have used any of the prohibited medications in that time.

\section{Treatments}

Patients were randomised 1:1 to the following treatments: once-daily UMEC metered dose $62.5 \mu \mathrm{g}$ (delivery dose $55 \mu \mathrm{g}$ ) via the Ellipta DPI (a multi-dose inhaler) or once-daily GLYCO metered dose $50 \mu \mathrm{g}$ (delivery dose $44 \mu \mathrm{g}$ ) via Breezhaler DPI (a single-dose inhaler). 
Use of inhaled corticosteroids (ICS) at a dose of $\leqslant 1000 \mu \mathrm{g} \cdot$ day $^{-1}$ of fluticasone propionate or equivalent was permitted during the study under the following conditions. Any ICS product alone could not be initiated or discontinued within 30 days before visit 1 . Patients taking ICS/long-acting $\beta_{2}$ agonist (LABA) could switch to ICS, provided the switch occurred $48 \mathrm{~h}$ before visit 1, if the LABA component was salmeterol or formoterol, or 14 days if the LABA component was vilanterol. Also, antibiotics and systemic corticosteroids for short-term treatment ( $\leqslant 14$ days) of COPD exacerbations were permitted during the study. All patients were provided with albuterol/salbutamol for as-needed rescue medication.

\section{Outcomes and assessments}

The primary endpoint was trough FEV1 on day 85, defined as the mean of the FEV 1 values obtained 23 and $24 \mathrm{~h}$ after the last study dose, in the per-protocol population.

Lung-function efficacy endpoints were also assessed in the intent-to-treat (ITT) population. These included trough FEV1 and trough FVC at days 2, 28, 56, 84 and 85. A subset of patients completed 24-h spirometry on day 1 and day $84(24 \mathrm{H}$ population) and the resultant outcomes were: weighted mean FEV1, serial FEV 1 and time to onset on day 1, defined as the first time to an increase $\geqslant 100 \mathrm{~mL}$ above baseline FEV1 in the first $6 \mathrm{~h}$ of spirometry.

Breathlessness was assessed using the transitional dyspnoea index (TDI) focal score and the proportion of responders at days 28, 56 and 84 (a TDI response was defined as a $\geqslant 1$ unit TDI focal score [22]). HRQoL was assessed using the St George's Respiratory Questionnaire (SGRQ) and the COPD Assessment Test (CAT). The outcomes analysed were SGRQ score and the proportion of responders (an SGRQ response was defined as an SGRQ total score of 4 units below baseline or lower [23]) and CAT score and the proportion of responders (a CAT response for an individual was defined as a CAT score 2 units below baseline or lower [24]). Rescue-medication use (mean puffs $\cdot$ day $^{-1}$ and percentage of rescue-free days) over the study duration was also assessed. All non-lung-function endpoints were assessed in the ITT population, with responders defined according to the published minimal clinically important differences (MCIDs).

The ITT population was used to measure "ease of use" of the Ellipta and Breezhaler DPIs at days 28 and 84. Further inhaler assessments were also performed in a subset of the ITT population, the inhaler error population. The proportion of patients making at least one overall error and one critical error with the Ellipta or the Breezhaler DPI at days 1,28 and 84 was measured.

A post hoc exploratory analysis was completed on the proportion of patients that achieved a $\geqslant 100 \mathrm{~mL}$ increase in trough FEV1 (MCID [25]) on each day of the study (ITT population). Trough FEV1 at day 85 was also analysed by the following subgroups: Global Initiative for Chronic Obstructive Lung Disease (GOLD) grade, GOLD group and ICS use.

Safety assessments included adverse events and COPD exacerbations in the ITT population. An exacerbation was defined as any acute worsening of COPD symptoms that necessitated the use of systemic corticosteroids, antibiotics and/or emergency treatment or hospitalisation.

\section{Statistical analysis}

The ITT population comprised all patients randomised to treatment who received at least one dose of randomised study medication. The per-protocol population comprised all patients in the ITT population who were not identified as full protocol deviators. The per-protocol population was used for the primary comparison of the primary endpoint as patients who deviated from the protocol may tend to bias the results towards equivalence, which could make a truly inferior treatment appear to be non-inferior [26]. In an effort to avoid bias, protocol deviations were defined, reviewed and determined by the study team who did not know which treatment a subject was assigned to.

As this study was not designed to determine the non-inferiority of other endpoints, the ITT population was used for these subsequent analyses because it adheres to the randomisation procedures and is generally conservative [27].

Two further subsets of the ITT population were also analysed. The $24 \mathrm{H}$ population was comprised of patients in the ITT population on whom 24-h spirometry was performed. The inhaler error population was comprised of a subset of patients who completed the inhaler error assessment.

The sample size calculations used a one-sided $2.5 \%$ significance level and an estimate of residual standard deviation for trough FEV1 of $220 \mathrm{~mL}$. A study with trough FEV1 data from 816 subjects would have $90 \%$ power to determine the non-inferiority of UMEC to GLYCO at day 85, with a non-inferiority margin of $50 \mathrm{~mL}$ and assuming the true mean treatment difference to be $0 \mathrm{~mL}$. Assuming a $10 \%$ withdrawal rate and a $10 \%$ exclusion rate from the per-protocol population, 1006 patients were necessary for randomisation. 
UMEC could be considered non-inferior to GLYCO if the lower bound 95\% confidence interval around the estimated treatment difference was above $-50 \mathrm{~mL}$ in the per-protocol population.

The sample size for the $24 \mathrm{H}$ population was chosen to provide sufficient data to allow the descriptive analysis of lung-function response over $24 \mathrm{~h}$. Similarly, the sample size of the inhaler error population was chosen to allow descriptive analysis of the errors made with both inhalers. Neither of these populations was used for formal statistical tests.

Superiority could be demonstrated if the treatment difference on trough FEV1 at day 85 in the per-protocol population was statistically significant at the $5 \%$ level (two-sided) and the corresponding $95 \%$ confidence interval was above $0 \mathrm{~mL}$. The statistical significance of all analyses could be inferred only if superiority was demonstrated.

If non-inferiority was demonstrated, but not superiority, then the statistical significance of other lung-function endpoints could not be inferred. However, the primary endpoint of trough FEV 1 was considered to be independent of the non-lung function endpoints and, therefore, all non-lung-function endpoints (i.e. TDI, SGRQ, CAT and rescue-medication use) could be termed statistically significant if $\mathrm{p}<0.05$.

All statistical analyses were completed using a Harmonisation for Analysis and Reporting environment with SAS version 9.3 (SAS Institute Inc., Cary, NC, USA) or a later release.

\section{Results}

\section{Patients}

Of the 1352 patients enrolled, 1037 were randomly assigned to treatment (figure 1). The ITT population comprised 1034 patients and the per-protocol population 986 patients (UMEC, n=494; GLYCO, n=492).

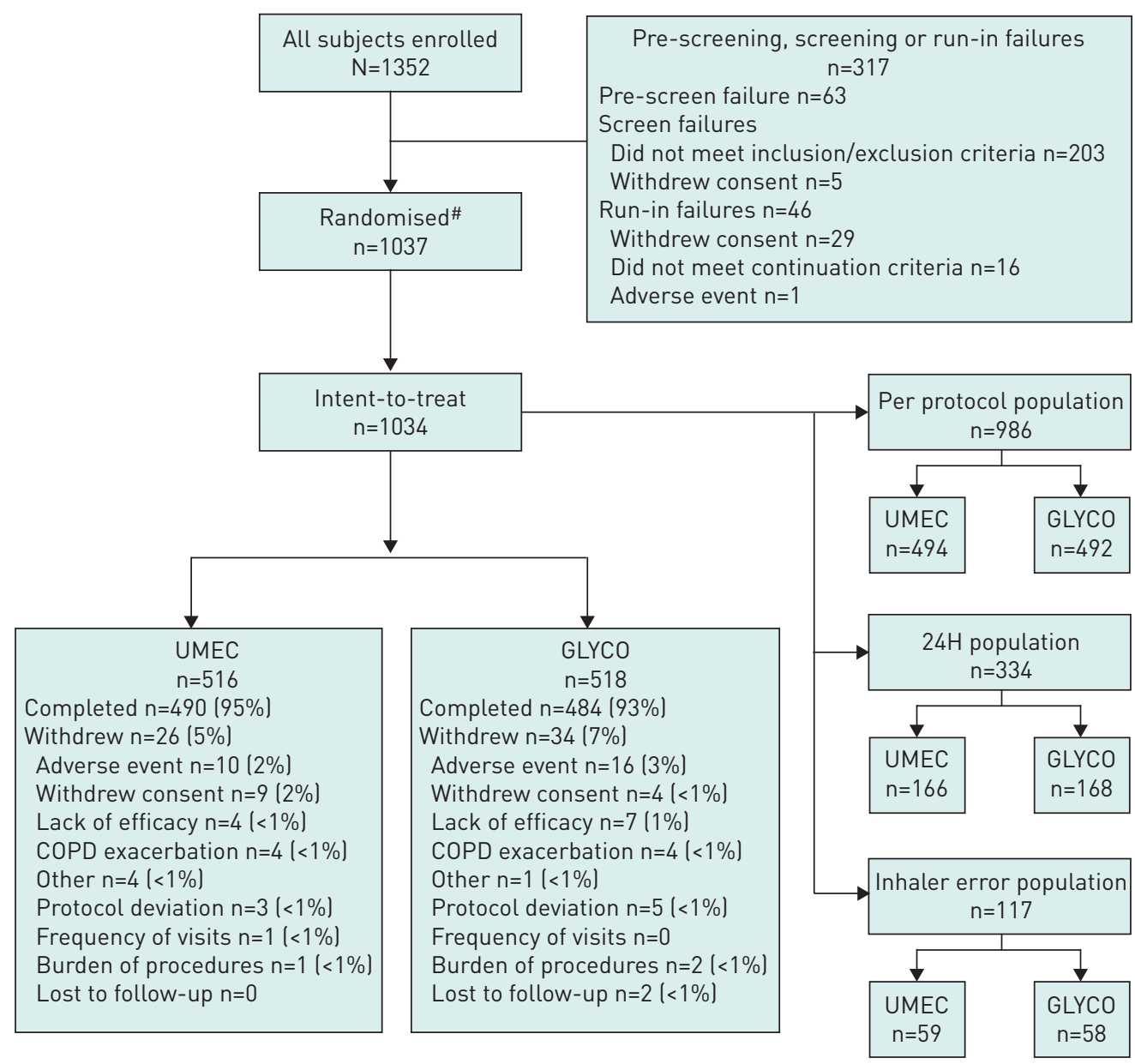

FIGURE 1 Consolidated Standards of Reporting Trials (CONSORT) diagram of patients enrolled in the study. \#: Two pre-screen/screen failures were erroneously randomised, one to each study arm. They were subsequently excluded from the intent-to-treat (ITT) population, as they did not receive a dose of study medication. A further patient was excluded from the ITT population due to a protocol deviation. UMEC: umeclidinium; GLYCO: glycopyrronium; $24 \mathrm{H}$ : subset of patients who completed 24-h spirometry on day 1 and day 84; COPD: chronic obstructive pulmonary disease. 
The $24 \mathrm{H}$ population included 334 patients and the inhaler error population 117 patients (figure 1). Baseline demographics and clinical characteristics of the treatment groups were similar (table 1). The majority of patients in the ITT population were male and the mean \% predicted FEV 1 was $50.4 \%$ and $50.6 \%$ in the UMEC and GLYCO groups, respectively, indicative of moderate-to-severe airflow obstruction in both groups (table 1).

\section{Efficacy}

For the primary endpoint of trough $\mathrm{FEV}_{1}$ at day 85 in the per-protocol population, UMEC was non-inferior to GLYCO because the lower bound for the $95 \%$ confidence interval for the comparison was above the non-inferiority margin of $-50 \mathrm{~mL}$ (treatment difference for UMEC versus GLYCO=24 $\mathrm{mL}, 95 \%$ CI: -5-54; figure 2). However, superiority of UMEC versus GLYCO was not observed as the bounds of the $95 \%$ confidence interval crossed $0(-5-54)$. The least squares mean \pm SE change from baseline of trough FEV 1 at day 85 was $123 \pm 10.5 \mathrm{~mL}$ for UMEC versus $99 \pm 10.5 \mathrm{~mL}$ for GLYCO, also showing that UMEC and GLYCO provided similar improvements in trough FEV1. The results for trough FEV1 in the per-protocol population were similar for both treatment groups over the duration of the study (figure 2).

When trough FEV 1 was analysed in the ITT population, similar results in the least squares mean change from baseline were observed (table 2). Analysis of trough FVC also demonstrated the treatment with UMEC and GLYCO resulted in similar improvements (ITT population; table 2).

In the $24 \mathrm{H}$ population, both treatments produced similar positive outcomes in $0-24 \mathrm{~h}$ weighted mean FEV1 (table 2) and also in $0-12 \mathrm{~h}$ and $12-24 \mathrm{~h}$ weighted mean FEV1 (data not shown). The serial FEV1 profiles of both treatments on days 1 and 84 were also similar (data not shown). The median time to reach $\geqslant 100 \mathrm{~mL} \mathrm{FEV} 1$ above baseline during the $0-6 \mathrm{~h}$ post-dose period on day 1 was longer in the UMEC group (30 min) versus the GLYCO group (15 $\mathrm{min}$ ).

TABLE 1 Baseline demographics and clinical characteristics (intent-to-treat population)

\begin{tabular}{|c|c|c|}
\hline & UMEC & GLYCO \\
\hline Patients n & 516 & 518 \\
\hline Age years & $64.1 \pm 8.4$ & $64.0 \pm 8.3$ \\
\hline Male & 355 (69) & $350(68)$ \\
\hline Current smoker & $242(47)$ & $249(48)$ \\
\hline Smoking pack-years $\#$ & $41.5 \pm 24.1$ & $42.0 \pm 23.4$ \\
\hline ICS use at screening & $239(46)$ & $255(49)$ \\
\hline Pre-salbutamol FEV $1 L^{\pi}$ & $1.350 \pm 0.461$ & $1.333 \pm 0.441$ \\
\hline Post-salbutamol FEV1 L & $1.493 \pm 0.462$ & $1.490 \pm 0.450$ \\
\hline Post-salbutamol FEV 1 /FVC & $48.9 \pm 9.7$ & $48.1 \pm 10.1$ \\
\hline Percent predicted FEV 1 & $50.4 \pm 10.6$ & $50.6 \pm 10.5$ \\
\hline Percent reversibility to salbutamol" & $12.5 \pm 13.6$ & $13.8 \pm 14.3$ \\
\hline Reversible to salbutamol $^{+}$ & $155(30)$ & 179 (35) \\
\hline \multicolumn{3}{|l|}{ GOLD grade (\% predicted FEV 1 ) } \\
\hline Grade $1(\geqslant 80 \%)$ & 0 & $1(<1)$ \\
\hline Grade 2 ( $\geqslant 50 \%$ to $<80 \%$ ) & $282(55)$ & 276 (53) \\
\hline Grade $3(\geqslant 30 \%$ to $<50 \%)$ & $233(45)$ & $240(46)$ \\
\hline Grade $4(<30 \%)$ & $1(<1)$ & $1(<1)$ \\
\hline \multicolumn{3}{|l|}{ GOLD category A-D, using mMRC } \\
\hline B (low risk, more symptoms) & $243(47)$ & $238(46)$ \\
\hline D (high risk, more symptoms) & $273(53)$ & $280(54)$ \\
\hline \multicolumn{3}{|c|}{ Patients with $\geqslant 1$ COPD exacerbation in the 12 months prior to enrolment } \\
\hline Managed without oral/systemic corticosteroids and/or antibiotics & 35 (7) & $28(5)$ \\
\hline $\begin{array}{l}\text { Required oral/systemic corticosteroids and/or antibiotics } \\
\text { (not involving hospitalisation) }\end{array}$ & $112(22)$ & $116(22)$ \\
\hline Required hospitalisation & $52(10)$ & $56(11)$ \\
\hline \multicolumn{3}{|c|}{ 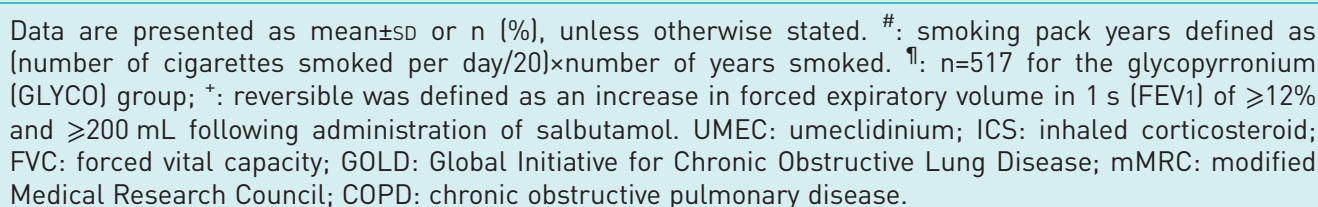 } \\
\hline
\end{tabular}




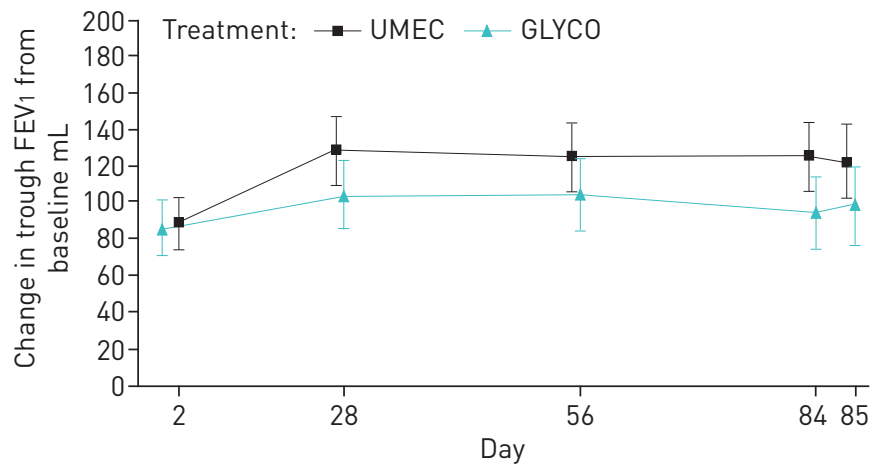

FIGURE 2 Change from baseline in trough forced expiratory volume in $1 \mathrm{~s}$ (FEV 1 ) (per-protocol population). No inferences can be made from the above comparisons at days $2,28,56$ and 84 as this was a lung-function endpoint and the superiority criterion at day 85 was not reached. Data are presented as least squares mean $\pm 95 \% \mathrm{Cl}$. UMEC: umeclidinium; GLYCO: glycopyrronium.

The TDI scores obtained with UMEC and GLYCO at each timepoint were similar (all p $>0.05$; table 3). Also, the improvements in SRGQ score from baseline were similar with the two treatments $(p>0.05)$, as were the improvements in CAT score ( $\mathrm{p}>0.05$; table 3 ). By day 84 , a clinically important improvement in TDI $(\geqslant 1$ unit), and SGRQ ( $\geqslant 4$ unit improvement from baseline) was observed in both groups; however, neither treatment improved CAT score above the 2 unit MCID [24] (table 3). For each responder category (TDI, SGRQ and CAT) analysed, the proportions of responders with UMEC and GLYCO treatments were similar at days 28 and 84 (TDI responders: 51-54\%; SGRQ responders: 43-46\%; CAT responders: 44-48\%; table 3).

Rescue salbutamol use over the 12-week study was similar in the UMEC and GLYCO treatment arms (least squares mean \pm SE change from baseline: $-0.8 \pm 0.06$ versus $-0.8 \pm 0.07$ puffs $\cdot$ day $^{-1}$, respectively; difference $(95 \%$ CI): $-0.1(-0.2-0.1)$ puffs $\cdot$ day $\left.^{-1} ; \mathrm{p}=0.556\right)$. The percentage of rescue medication-free days reported in both treatment arms had skewed distributions; however, there was no treatment difference between the percentage of rescue-free days for UMEC and GLYCO analysed non-parametrically (UMEC $=488$ patients, GLYCO $=485$ patients; estimated median $(95 \% \mathrm{CI})$ treatment difference $0(-0.3,0.0) ; \mathrm{p}=0.871)$.

\section{Inhaler assessments}

The majority of patients found their assigned inhaler "easy" or "very easy" to use. At both timepoints (days 28 and 84), a larger percentage of patients that used the Ellipta DPI (61\% and 68\%, respectively) found it "very easy" to use compared with patients using the Breezhaler DPI (50\% at both timepoints). When assessing

\section{TABLE 2 Changes in lung function in the intent-to-treat population and in the $24 \mathrm{H}$ population}

\begin{tabular}{|c|c|c|c|}
\hline & UMEC & GLYCO & Difference UMEC versus GLYCO $(95 \% \mathrm{Cl})^{\#}$ \\
\hline Intent-to-treat population $\mathbf{n}$ & 516 & 518 & \\
\hline \multicolumn{4}{|l|}{ Trough $\mathrm{FEV}_{1}{ }^{\uparrow}$ day 85} \\
\hline Patients with analysable data & 486 & 481 & \\
\hline Change from baseline $\mathrm{mL}$ & $126 \pm 10$ & $93 \pm 10$ & $33(5-61)$ \\
\hline \multicolumn{4}{|l|}{ Trough FVC ${ }^{\text {Il }}$ day 85} \\
\hline Patients with analysable data & 486 & 481 & \\
\hline Change from baseline $\mathrm{mL}$ & $157 \pm 16$ & $143 \pm 16$ & $14(-31-59)$ \\
\hline $24 \mathrm{H}$ population $n$ & 166 & 168 & \\
\hline \multicolumn{4}{|l|}{ Weighted mean FEV $10-24 \mathrm{~h}^{+}$day 1} \\
\hline Patients with analysable data & 166 & 166 & \\
\hline Change from baseline $\mathrm{mL}$ & $61 \pm 12$ & $84 \pm 12$ & $-23(-57-10)$ \\
\hline \multicolumn{4}{|c|}{ Weighted mean FEV $10-24 \mathrm{~h}^{+}$day 84} \\
\hline Patients with analysable data & 155 & 159 & \\
\hline Change from baseline $\mathrm{mL}$ & $62 \pm 18$ & $67 \pm 17$ & $-5(-54-44)$ \\
\hline
\end{tabular}

Data are presented as least-square mean $\pm \mathrm{SE}$, or $\mathrm{n}$, unless otherwise stated. ${ }^{\#}$ : no inferences can be made from the above comparisons because this was a lung-function endpoint and the superiority criterion was not reached; ": analysis performed using a repeated measures model with covariates of treatment, baseline forced expiratory volume in $1 \mathrm{~s}$ (FEV 1 ) or forced vital capacity (FVC) (mean of the values measured at $30 \mathrm{~min}$ and $5 \mathrm{~min}$ pre-dose on day 1), centre group, $24 \mathrm{H}$ subset flag, day, day by baseline and day by treatment interactions; ${ }^{+}$: analysis performed using a mixed-model repeated measures model including covariates of baseline $F E V 1$, centre group, $24 \mathrm{H}$ subset flag, day, treatment, day by baseline interaction and day by treatment interaction; $24 \mathrm{H}$ : subset of patients who completed 24-h spirometry on day 1 and day 84; GLYCO: glycopyrronium; UMEC: umeclidinium. 
TABLE 3 Changes in TDI score, SGRQ score and CAT score (intent-to-treat population)

UMEC

GLYCO

\section{Patients}

\section{TDI score}

Day $28^{\#}$

Patients with analysable data

Change from baseline

\section{Day $56^{\#}$}

Patients with analysable data

Change from baseline

\section{Day $\mathbf{8 4}{ }^{\#}$}

Patients with analysable data

Change from baseline

\section{TDI responders}

Day 28 ?

Total patients

Responders

Day 56 ?

Total patients

Responders

Day $84^{\text {? }}$

Total patients

Responders

\section{SGRQ total score}

Day $28^{\#}$

Patients with analysable data

Change from baseline

\section{Day $\mathbf{8 4}$}

Patients with analysable data

Change from baseline

\section{SGRQ responders}

Day 28 ๆ

Total patients

Responders

Day $84^{\text {? }}$

Total patients

Responders

\section{CAT score}

Day $28^{\#}$

Patients with analysable data

Change from baseline

\section{Day $\mathbf{8 4}{ }^{\#}$}

Patients with analysable data

Change from baseline

\section{CAT responders}

\section{Day 28 ?}

Total patients

Responders

\section{Day 84 ?}

Total patients

Responders
518

516

502

$1.36 \pm 0.116$

497

$1.31 \pm 0.116$

492

497

$1.67 \pm 0.117$

$1.48 \pm 0.118$

491

486

$1.74 \pm 0.116$

$1.66 \pm 0.116$

515

$266[52]$

514

$260(51)$

$515 \quad 515$

$289(56)$

249 (48)

515

$280(54)$

515

$268(52)$

491

$-4.16 \pm 0.467$

479

$-4.13 \pm 0.472$

477

$-4.75 \pm 0.545$

466

$-4.11 \pm 0.551$

504

218 (43)

508

$221(44)$

503

$-1.29 \pm 0.226$

494

$-1.70 \pm 0.254$

516

227 (44)

516

$237(46)$
497

$224(45)$

503

$230(46)$

500

$-1.27 \pm 0.226$

488

$-1.19 \pm 0.256$

518

251 (48)

518

249 (48)
Difference or odds ratio UMEC versus GLYCO $(95 \%$ CI)

\section{Difference}

$0.05(-0.27,0.38) ; p=0.743$

$0.19(-0.14,0.51) ; p=0.261$

$0.08(-0.24,0.40) ; p=0.636$

Odds ratio

$1.05(0.82,1.36) ; p=0.694$

$1.44(1.11,1.88) ; p=0.007^{+}$

$1.12(0.87,1.46) ; p=0.379$

Difference

$-0.03(-1.33,-1.28) ; p=0.970$

$-0.64(-2.17,0.88) ; p=0.406$

Odds ratio

$0.94(0.73,1.22) ; p=0.660$

$0.92(0.72,1.19) ; p=0.541$

Difference

$-0.02(-0.65,0.61) ; p=0.949$

$-0.51(-1.22,0.19) ; p=0.156$

Odds Ratio

$0.85(0.66,1.09) ; p=0.199$

$0.94(0.73,1.21) ; p=0.630$

Data are presented as $\mathrm{n}$, least squares mean \pm SE or $\mathrm{n}(\%)$, unless otherwise stated. Differences and odd ratios are umeclidinium (UMEC) versus glycopyrronium (GLYCO). " : analysis performed using a mixed-model repeated measures with covariates of treatment, baseline dyspnoea index score or baseline St George's Respiratory Questionnaire (SGRQ) or chronic obstructive pulmonary disease assessment test (CAT) total score (scores recorded on day 1), centre group, $24 \mathrm{H}$ subset flag, day, day by baseline and day by treatment interactions. ": analysis performed using a separate logistic regression model at each visit with covariates of treatment, baseline dyspnoea index Score or SGRQ or CAT Score at baseline, centre group and $24 \mathrm{H}$ subset flag. Transition dyspnoea index (TDI) response was defined as patients who had a $\geqslant 1$ unit TDI focal score. SGRQ response was defined as an SGRQ total score of 4 units below baseline (scores recorded on day 1) or lower. CAT response within a patient was defined as a CAT score 2 units below baseline or lower. ${ }^{+}$: inference of statistical significance was drawn from $p$-values for treatment comparisons on these non-lung-function related endpoints and they were termed statistically significant if $p<0.05$. $24 \mathrm{H}$ : subset of patients who completed 24-h spirometry on day 1 and day 84 . 
overall and critical errors in the inhaler error population, the number of errors was low and decreased over the course of the study for the Ellipta DPI and the Breezhaler DPI (supplementary table s2).

\section{Post hoc analysis}

From day 28 onwards, treatment with UMEC appeared to result in a greater number of trough FEV1 responders $(\geqslant 100 \mathrm{~mL}$ improvement from baseline in the ITT population) versus treatment with GLYCO; however, no inferences can be made because it was a lung-function endpoint and the superiority criterion was not reached (figure 3). When trough FEV1 at day 85 was analysed by subgroup (GOLD grade 1-2, GOLD grade 3-4, GOLD group B, GOLD group D and ICS use), differences appeared to favour UMEC; however, no statistical inferences can be made about these results (supplementary table s3).

\section{Safety}

Adverse events profiles were similar for the two treatment groups (table 4). Four deaths occurred on-treatment during the study, two in each treatment group (UMEC, <1\%: one due to sudden cardiac death and one due to sudden death; GLYCO, $<1 \%$ : one due to acute coronary syndrome and one to laryngeal cancer), none of which was considered related to the study drug by the reporting investigator.

The majority of patients did not experience a COPD exacerbation during the study (UMEC, 91\%; GLYCO, 90\%). The total number of exacerbations in the UMEC group was 52, experienced by 47 (9\%) patients, and 60 in the GLYCO group, experienced by $51(10 \%)$ patients. The majority of these were treated with oral/systemic corticosteroids and resolved during the study period.

\section{Discussion}

This study demonstrated non-inferiority between once-daily UMEC $62.5 \mu \mathrm{g}$ delivered by the Ellipta DPI and GLYCO $50 \mu \mathrm{g}$ delivered by the Breezhaler DPI for trough FEV1 at day 85 in the per-protocol population. Clinically important lung-function improvements (trough $\mathrm{FEV}_{1} \geqslant 100 \mathrm{~mL}$ [25]) achieved with both UMEC and GLYCO were accompanied by clinically important improvements in other endpoints TDI $(\geqslant 1$ unit $[28,29])$, SGRQ (improvement of $\geqslant 4$ units [23]) and at the population level for CAT with UMEC only ( $\geqslant 1.6$ units [30]). While the MCID for CAT is a change of 2 units in an individual [24], the threshold is smaller for a population at 1.6 units [30]. Treatment with UMEC improved the population mean CAT score in this study beyond this threshold. Overall, both treatments, therefore, not only improved lung function but also improved symptoms and HRQoL within the study.

There was a difference in time to onset favouring GLYCO versus UMEC (15 versus 30 min, respectively) on day 1; however, the magnitude of the difference is small and the study was not designed to show statistical differences between arms in terms of time to onset. However, the difference in time to onset was small and the magnitude of lung-function improvements on day 1 after the first 30 min was similar between the two treatments. Serial lung-function improvements were similar at all timepoints at other days of testing. A similar transient treatment difference has been reported between GLYCO and TIO in a previous study; however, it was evident only at day 1 of that study [10]. The difference was not maintained with repeat dosing, as there was no difference at week 12 [10].

This study extends results of previous studies that investigated UMEC [16, 17] or GLYCO [19, 20, 31], by comparing these two LAMAs within a single randomised trial. The efficacy and safety of once-daily UMEC $62.5 \mu \mathrm{g}$ and once-daily GLYCO $50 \mu \mathrm{g}$ were found to be similar. It should be noted that this study

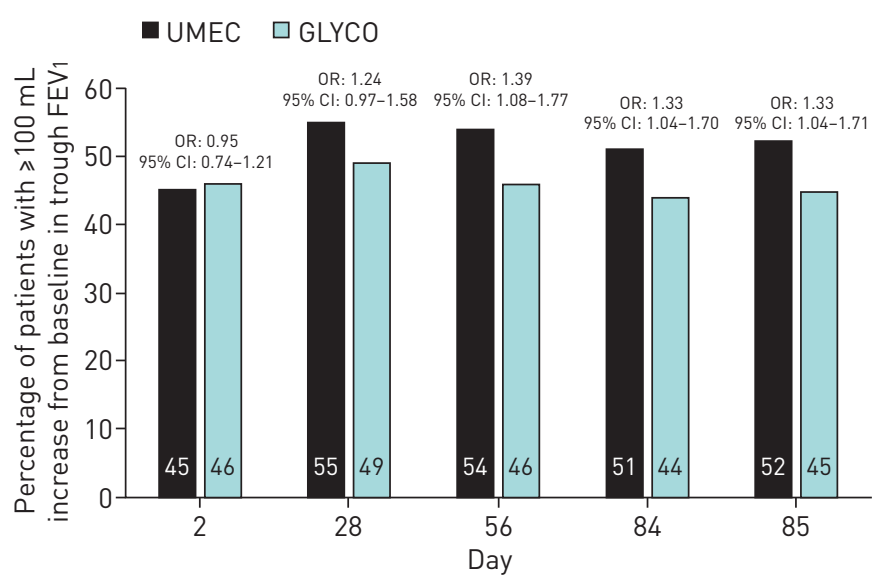

FIGURE 3 The proportion of patients with $a \geqslant 100 \mathrm{~mL}$ increase in trough forced expiratory volume in $1 \mathrm{~s}$ (FEV 1 ] from baseline lintent-to-treat population, post hoc analysis). No inferences can be made from the above comparisons as this was a lung-function endpoint and the superiority criterion was not reached. GLYCO: glycopyrronium; UMEC: umeclidinium. 


\begin{tabular}{|c|c|c|}
\hline & UMEC & GLYCO \\
\hline Patients $\mathrm{n}$ & 516 & 518 \\
\hline \multicolumn{3}{|l|}{ Adverse event category } \\
\hline Any on-treatment adverse event & 193 (37) & $188(36)$ \\
\hline Any on-treatment drug-related adverse event ${ }^{\#}$ & $17(3)$ & $18(3)$ \\
\hline Any on-treatment non-fatal SAE & $15(3)$ & $13(3)$ \\
\hline Any on-treatment non-fatal drug-related $S A E^{\#}$ & $1(<1\}$ & 0 \\
\hline Any on-treatment fatal SAE & $2(<1)$ & $2(<1)$ \\
\hline \multicolumn{3}{|c|}{$\begin{array}{l}\text { On-treatment } \mathrm{AE} \text { reported by } \geqslant 3 \% \text { of subjects in either } \\
\text { group by preferred term }\end{array}$} \\
\hline Headache & $42(8)$ & $51(10)$ \\
\hline Nasopharyngitis & $42(8)$ & $39(8)$ \\
\hline \multicolumn{3}{|c|}{ Non-fatal SAE reported by $\geqslant 2$ patients by preferred term } \\
\hline COPD" & $7(1)$ & $5(<1)$ \\
\hline Pneumonia & $2(<1)$ & 0 \\
\hline Atrial fibrillation & $1(<1)$ & $1(<1)$ \\
\hline \multicolumn{3}{|l|}{$A E$ of special interest by preferred term } \\
\hline Cardiovascular events & $12(2)$ & $11(2)$ \\
\hline Pneumonia & $4(<1)$ & $4(<1)$ \\
\hline LRTI excluding pneumonia & $5(<1)$ & $6(1)$ \\
\hline
\end{tabular}

Data are presented as $\mathrm{n}(\%)$, unless otherwise stated. *: drug-related adverse events (AEs) or severe adverse events (SAEs) were defined by the study investigator; ${ }^{7}$ : chronic obstructive pulmonary disease (COPD) was listed as an AE by the participating investigator and was likely due to a worsening of the underlying condition. GLYCO: glycopyrronium; UMEC: umeclidinium; LRTI: lower respiratory tract infection.

used a once-daily GLYCO delivered dose of $44 \mu \mathrm{g}$, the approved dose in the EU [3]. In the USA, GLYCO is approved for administration of $12.5 \mu \mathrm{g}$ twice-daily delivered dose [4] and the findings of this study cannot be generalised to alternative GLYCO dosing regimens.

The main limitation of this study was its open-label design, which may have resulted in some degree of bias as patients and study site investigators were aware of the study treatments used. The study was not designed to assess differences in exacerbations between treatments; further studies would be needed to investigate this. Additionally, as all patients were trained on their assigned device, it is likely that inhaler errors were minimised and not reflective of real-world use.

In conclusion, once-daily treatment with UMEC $62.5 \mu \mathrm{g}$ was non-inferior to once-daily GLYCO $50 \mu \mathrm{g}$ in patients with moderate-to-severe COPD. Patient-reported outcomes and safety profiles were similar for both treatments.

\section{Acknowledgements}

This study (201315) was funded by GlaxoSmithKline. Chris Kalberg (GlaxoSmithKline, Respiratory and ImmunoInflammation, Research Triangle Park, NC, USA) provided assistance with writing the final study report and the outline of this manuscript. Palvi Shah (GlaxoSmithKline, Respiratory Research and Development, Middlesex, UK) provided substantial contributions to the study data analysis, data interpretation, and preparation of the manuscript. Editorial assistance (in the form of writing assistance, assembling tables and figures, collating author comments, grammatical editing and referencing) was provided by Gillian Groeger and Joanne Ashworth, of Fishawack Indicia Ltd, Oxford and Knutsford, UK, and was funded by GlaxoSmithKline.

\section{References}

1 Global Initiative for Chronic Obstructive Lung Disease (GOLD) 2015. www.goldcopd.org/ Date last updated: 2015. Date last accessed: August 10, 2015.

2 Boehringer-Ingelheim. SPIRIVA. Prescribing Information. http://bidocs.boehringer-ingelheim.com/BIWebAccess/ ViewServlet.ser?docBase=renetnt\&folderPath=/Prescribing+Information/PIs/Spiriva/Spiriva.pdf Date last updated: 2014. Date last accessed: September 3, 2015.

3 Novartis. Seebri Breezhaler. Summary of product characteristics. www.ema.europa.eu/docs/en_GB/document_library/ EPAR_-_Product_Information/human/002430/WC500133769.pdf Date last updated: 2015. Date last accessed: September 5, 2015.

4 Novartis. Seebri Neohaler. Prescribing information. www.accessdata.fda.gov/drugsatfda_docs/label/2015/207923lbl. pdf Date last updated: 2015. Date last accessed: November 6, 2015.

5 GlaxoSmithKline. INCRUSE. Summary of product characteristics. www.ema.europa.eu/ema/index.jsp?curl=pages/ medicines/human/medicines/002809/human_med_001755.jsp\&mid=WC0b01ac058001d124 Date last updated: 2015. Date last accessed: September 3, 2015. 
6 GlaxoSmithKline. INCRUSE. Prescribing information. www.gsksource.com/pharma/content/dam/GlaxoSmithKline/ US/en/Prescribing_Information/Incruse_Ellipta/pdf/INCRUSE-ELLIPTA-PI-PIL.PDF Date last updated: 2014. Date last accessed: September 3, 2015.

7 AstraZeneca. Eklira Genuair. Summary of product characteristics. www.ema.europa.eu/docs/en_GB/document_library/ EPAR_-_Product_Information/human/002211/WC500132661.pdf Date last updated: 2015. Date last accessed: September 9, 2015 .

8 Almirall. Tudorza Pressair. Prescribing information. www.accessdata.fda.gov/drugsatfda_docs/label/2012/ 202450s000lbl.pdf Date last updated: 2012. Date last accessed: October 28, 2015.

9 Boehringer-Ingelheim. SPIRIVA. Summary of product characteristics. www.medicines.org.uk/emc/medicine/ 201341. Date last updated: 2014. Date last accessed: October 29, 2015.

10 Chapman KR, Beeh KM, Beier J, et al. A blinded evaluation of the efficacy and safety of glycopyrronium, a once-daily long-acting muscarinic antagonist, versus tiotropium, in patients with COPD: the GLOW5 study. BMC Pulm Med 2014; 14: 4.

11 Riario-Sforza GG, Ridolo E, Riario-Sforza E, et al. Glycopyrronium bromide for the treatment of chronic obstructive pulmonary disease. Expert Rev Respir Med 2015; 9: 23-33.

12 Cope S, Donohue JF, Jansen JP, et al. Comparative efficacy of long-acting bronchodilators for COPD: a network meta-analysis. Respir Res 2013; 14: 100

13 Karabis A, Lindner L, Mocarski M, et al. Comparative efficacy of aclidinium versus glycopyrronium and tiotropium, as maintenance treatment of moderate to severe COPD patients: a systematic review and network meta-analysis. Int J Chron Obstruct Pulmon Dis 2013; 8: 405-423.

14 Ismaila AS, Huisman EL, Punekar YS, et al. Comparative efficacy of long-acting muscarinic antagonist monotherapies in COPD: a systematic review and network meta-analysis. Int J Chron Obstruct Pulmon Dis 2015; 10: $2495-2517$

15 Segreti A, Calzetta L, Rogliani P, et al. Umeclidinium for the treatment of chronic obstructive pulmonary disease Expert Rev Respir Med 2014; 8: 665-671.

16 Donohue JF, Anzueto A, Brooks J, et al. A randomized, double-blind dose-ranging study of the novel LAMA GSK573719 in patients with COPD. Respir Med 2012; 106: 970-979.

17 Trivedi R, Richard N, Mehta R, et al. Umeclidinium in patients with COPD: a randomised, placebo-controlled study. Eur Respir J 2014; 43: 72-81.

18 D'Urzo A, Ferguson GT, van Noord JA, et al. Efficacy and safety of once-daily NVA237 in patients with moderate-to-severe COPD: the GLOW1 trial. Respir Res 2011; 12: 156.

19 Fogarty C, Hattersley H, Di Scala L, et al. Bronchodilatory effects of NVA237, a once daily long-acting muscarinic antagonist, in COPD patients. Respir Med 2011; 105: 337-342.

20 Kerwin E, Hebert J, Gallagher N, et al. Efficacy and safety of NVA237 versus placebo and tiotropium in patients with COPD: the GLOW2 study. Eur Respir J 2012; 40: 1106-1114.

21 Celli BR, MacNee W. Standards for the diagnosis and treatment of patients with COPD: a summary of the ATS/ ERS position paper. Eur Respir J 2004; 23: 932-946.

22 Mahler DA, Witek TJ Jr. The MCID of the transition dyspnea index is a total score of one unit. COPD 2005; 2 : 99-103.

23 Jones PW. St. George's Respiratory Questionnaire: MCID. COPD 2005; 2: 75-79.

24 Kon SS, Canavan JL, Jones SE, et al. Minimum clinically important difference for the COPD Assessment Test: a prospective analysis. Lancet Respir Med 2014; 2: 195-203.

25 Donohue JF. Minimal clinically important differences in COPD lung function. COPD 2005; 2: 111-124.

26 Snapinn SM. Noninferiority trials. Curr Control Trials Cardiovasc Med 2000; 1: 19-21.

27 Lewis JA, Machin D. Intention to treat: who should use ITT? Br J Cancer 1993; 68: 647-650.

28 Witek TJ Jr, Mahler DA. Meaningful effect size and patterns of response of the transition dyspnea index. J Clin Epidemiol 2003; 56: 248-255.

29 Witek TJ Jr, Mahler DA. Minimal important difference of the transition dyspnoea index in a multinational clinical trial. Eur Respir J 2003; 21: 267-272.

30 Jones PW, Harding G, Berry P, et al. Development and first validation of the COPD Assessment Test. Eur Respir J 2009; 34: 648-654.

31 Rennard S, Fogarty C, Reisner C, et al. Randomized study of the safety, pharmacokinetics, and bronchodilatory efficacy of a proprietary glycopyrronium metered-dose inhaler in study patients with chronic obstructive pulmonary disease. BMC Pulm Med 2014; 14: 118. 